\title{
Creativity Development in Science and Technology Education
}

\author{
Janchai Yingprayoon \\ \{janchai.yi@ssru.ac.th\} \\ International College, Suan Sunandha Rajabhat University, \\ Bangkok, Thailand ${ }^{1}$
}

\begin{abstract}
Creativity is a natural function of the mind that can be developed in several ways. The nature of innovative thinking and the characteristics of creative people can give ideas about how creativity can be developed. Creativity can be stimulated by using the SCAMPER method. The steps are substitute, combine, adapt, put to other use, eliminate, and rearrange. Four components of teachings in the classroom: observation, curiosity, sense of humor and creativity, are emphasized for the promotion of creativity in science and technology education.
\end{abstract}

Keywords: Creativity, International Patent, Creative people, Observation, Curiosity, SCAMPER.

\section{Introduction}

Creativity is a natural characteristic of the mind. It is not a question of talent. Everybody can be creative, or at least capable of developing creativity or doing something creative. In education, creativity has always been recognized. Increasingly, its importance in economic development, through ingenious use of resources for both commercial and social enterprise, and its crucial role in improving quality of life is also being acknowledged. Innovation and originality are seen as a defining characteristic of world culture in the twenty-first century, necessary for the future of humankind. And it has probably been as important as any human quality in changing history and reshaping the world. Rapid advancement in science and technology promotes further innovation and stimulates invention as individuals respond creatively to the challenges of their world. In recent decades, these observations have resulted in an increased interest in how creativity can be developed. If a nation is to survive in the midst of international competition, the most promising solution is for the country to encourage and support the identification and development of highly creative persons. Nations that learn how to encourage creativity in their citizens, especially amongst their children, will find themselves in a very advantageous position. There are well-known cases of creative people, whose ingenuity not being supported in their home countries, have migrated elsewhere for opportunties to contribute in original and significant ways. The table in Figure 1 shows the number of international patent applications from Asia-Pacific in 2017-2018 [1]. Patent applications could be a measure of the creative productivity of a nation. 


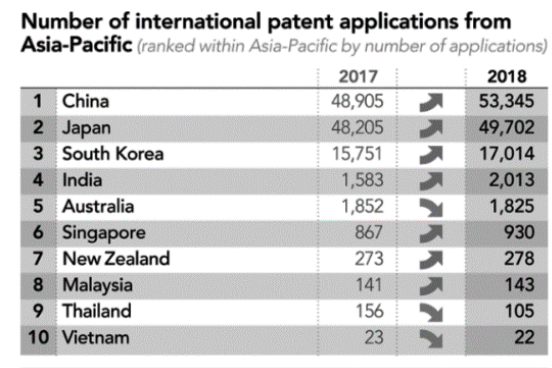

Fig. 1. The number of international patent applications from Asia-Pacific in 2017-2018 [1].

\section{What is Creativity?}

Creativity is a process of two stages. The first stage is to shake and mix ideas together and then chose from the variety of different possibilities that emerge. Secondly, one needs to synthesize and bring together the chosen elements in new and original ways. Thus creativity is seen in the product rather than in the process. Alternatively, creativity can be defined in terms of certain mental abilities. Indeed, there are probably hundreds of definitions of creativity, from light-hearted to serious, and simple to complicated.

But all of them seem to show that innovation or creativity is about making connections where none existed before. For example, consider Bett Nesmith, a woman who made millions by connecting painting with typing to make a liquid paper. Johan Gutenberg made a connection between a wine press and a coin punch to invent the printing press. Christopher Sholes made a connection between piano keys and a writing machine to invent the typewriter.

Teachers are in a good position to nurture the creativity demonstrated in children responding with originality and humour. Less conforming responses, alongside unpredictable behaviour, may also indicate creative thinking [2].

Conequences, however, of such imagination, especially in stricter home and school environments, can be an annoyance on the part of others toward the creative child, and a failure to recognize the gifts creative children bring to families and classrooms. Resourceful and creative children can become inspiring adults who can make a real difference with their creative problem-solving skills.

\section{Characteristics of Creative People}

Although every child is naturally creative to some degree, differences between children have been observed. Indications of greater creative aptitude in children include:

- greater self-sufficiency and independence in judgment

- more self-motivated and energetic

- more resourceful and adventurous, and more radical and self-assertive

- greater self-control and possibly more emotionally sensitive

- often more introverted but also demonstrating boldness

- a sense of humour 
- $\quad$ a preference for visual and kinesthetic over auditory means while learning

- a preference for sound in the background (rather than complete silence) while concentrating or studying

- $\quad$ study on their own rather than with classmates and adults.

- confidence

- curiosity

- idealism

Figure 2 shows some characteristics of creative people.

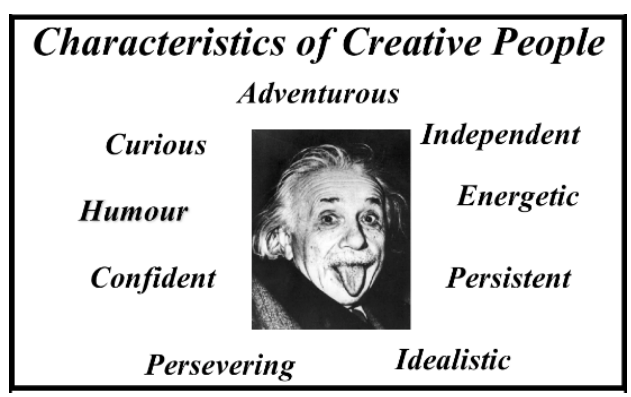

Fig. 2. Showing some characteristics of creative people.

Supporting the development of creativity in children is an important role for teachers and parents. Valuing the learning that can come through making mistakes and testing one's ideas as problems is an important part of that support. Permitting the child to try their ideas without fear and failure, and providing an accommodating environment for this experimentation is key. Adults should teach and encourage children need to see different perspectives and to generate several solutions as they problem-solve. Training to notice "cues" in the environment will help children generate a pool of possible solutions.

\section{How to develop students' creativity}

"Creativity is not a talent; it is a skill that can be learned. It empowers people by adding strength to their natural abilities which improves teamwork, productivity, and where appropriate - profits." Edward de Bono

Carefully planned strategies and techniques can help develop student creativity. Stimulating the pupils to think in new ways about an issue will assist all students, even those who do not demonstrate much natural creativity, to develop their imagination and ingenuity. The role of the teachers is very important for fostering creativity among students.

These are tips for parents and teachers to help children think creatively

- $\quad$ arouse curiosity

- challenge ideas

- $\quad$ avoid projects that can be completed in only one way (e.g. paint-by-numbers, assembly kits) 
- make a wide variety of materials available to children

- suggest options, but let the child make the final decision

- ask about the process of completing the project rather than guessing, possibly incorrectly, from an adult's point of view

- praise the effort, use of color and uniqueness rather than just the final product - the trip is more important than the destination

- encourage individual expression

- avoid regimented use of materials and adult-directed projects

A classroom full of samples of individual creativeness indicates that the teacher has given children choices, and has focused on the process rather than the product. According to Yew Kam Keong [3], there are five steps in developing creativity.

Knowledge: use all the five senses to acquire diverse knowledge

Thinking: think deeply

Incubation: relax and do something unrelated to the problem

Eureka!: I found it! Moment of Inspiration

Development: developing the ideas into useful and practical applications

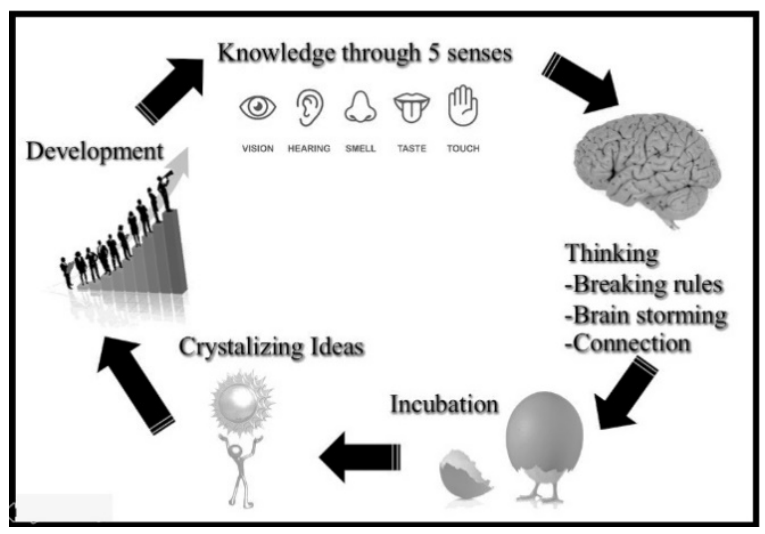

Fig. 3. Five steps for greater creativity [3].

The "SCAMPER" mnemonic for creativity is based on seven ideas suggested by Alex Osborn, a pioneer teacher of creativity, that were later arranged by Bob Eberle [4].

- Substitute something

- Combine it with something else

- Adapt something to it

- Modify or Magnify or Minify it

- Put it to some other use

- Eliminate something

- Reverse or Rearrange it

Figure 4 shows the SCAMPER method of Creativity Development. 


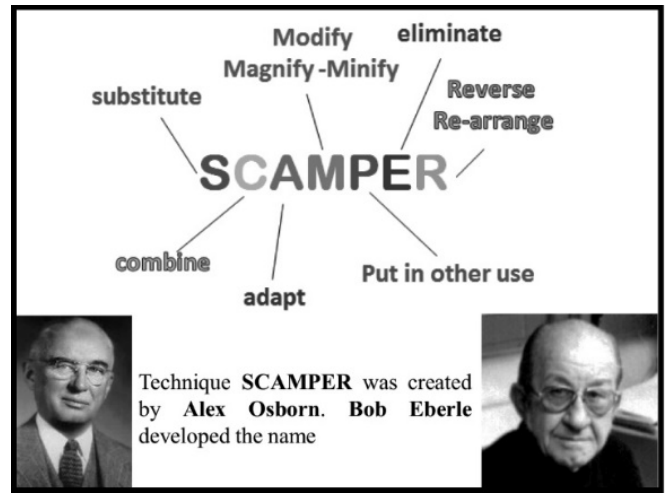

Fig. 4. Showing the SCAMPER method of Creativity Development.

To use SCAMPER:

1. Isolate the challenge or subject of interest.

2. Go through the SCAMPER stages about each step of the challenge or subject and see what new ideas emerge.

Below are some interesting samples of how to apply SCAMPER methods to develop better products or systems with creativity.

\section{SUBSTITUTE}

In many restaurants, the waiters asked customers what they would like to drink. Many times, the customers ask for "Anything" or "Whatever". In Singapore, the famous brand names of soft drinks have been substituted by the names "ANYTHING" and "WHATEVER" as shown in Figure 5. These two new soft drinks are very sellable.

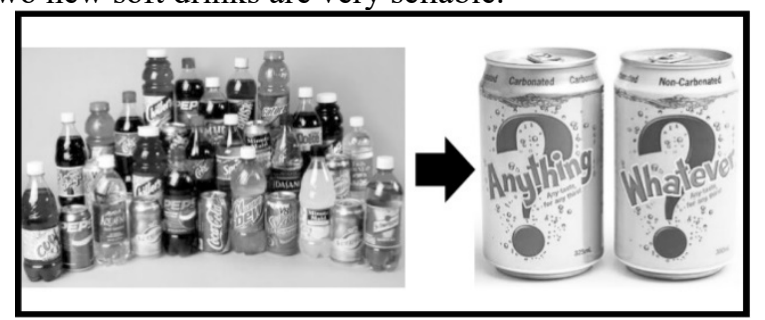

Fig. 5. Showing creative substitution of brands of soft drinks.

\section{COMBINE}

A washing machine and a toilet can be combined so that wastewater from washing can be used to flush the toilet. Figure 6 shows a combination of a washing machine and a toilet. 


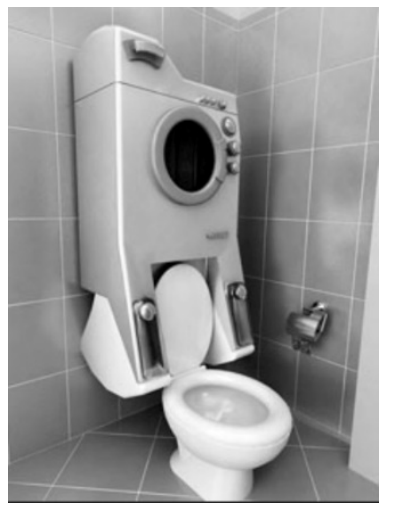

Fig. 6. A washing machine - toilet combination.

\begin{abstract}
ADAPT
The shape of a watermelon is normally round. But if we want to transport more watermelons at one time, we have to adapt or find a way to get watermelons into a cube shape. Or perhaps we want to sell watermelon to a hot country like Egypt, and so have to adapt the fruit into a Pyramid shape. Figure 7 shows the adapted shapes of watermelon.
\end{abstract}

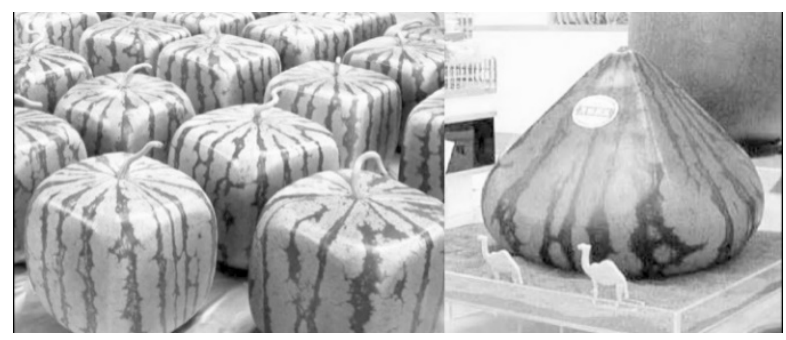

Fig. 7. Adapted shapes of watermelon.

\title{
MODIFY - MAGNIFY - MINIFY
}

In 1950, the size of a computer was the size of a room. After decades of development, we can now have a more powerful computer in the form of a palm-held tablet. Figure 8 shows the tremendous change in computer size. 


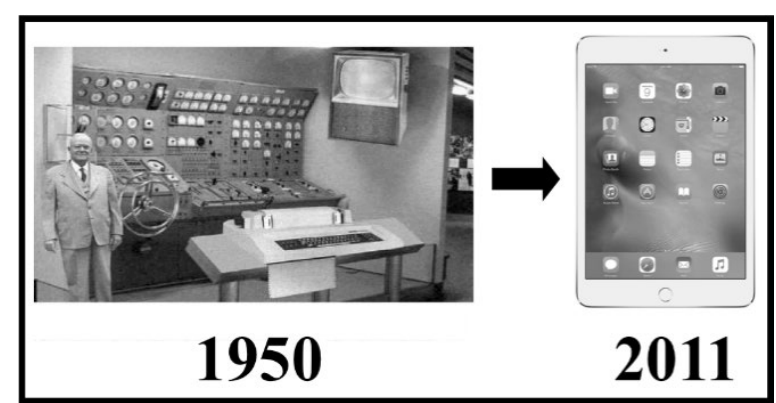

Fig. 8. Changes in computer size.

\section{PUT TO OTHER USE}

Normally the tea strainers are used for making tea. But in some areas, near large deserts, there are sand storms. Tea strainers can also be used as protective eye goggles, as shown in Figure 9.
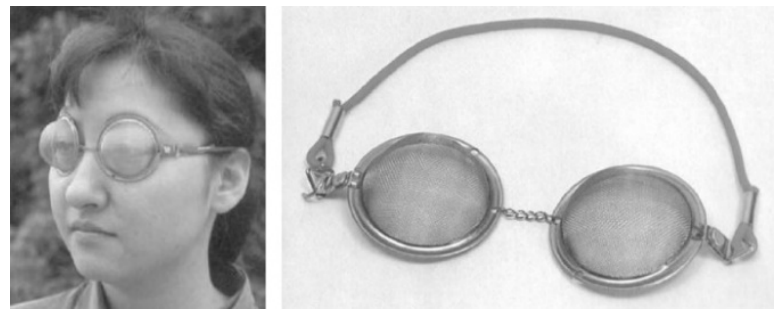

Fig. 9. Tea strainers as eye goggles.

\section{ELIMINATE}

We can improve the device or product by eliminating some aspect of the design in order to get a new creative product. Examples are contact lenses or a wireless microphone, as shown in Figure 10.

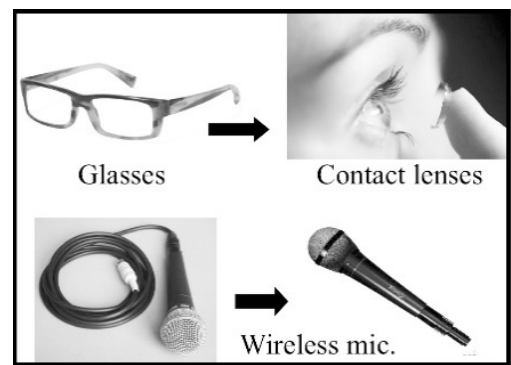

Fig. 10. Showing the elimination of unnecessary parts to get better products. 


\section{REARRANGE}

Every floor of Dubai's Moving Skyscraper "Dynamic Tower" can be rearranged or rotated to a position that is chosen by the people who live on that floor, as shown in Figure 11.

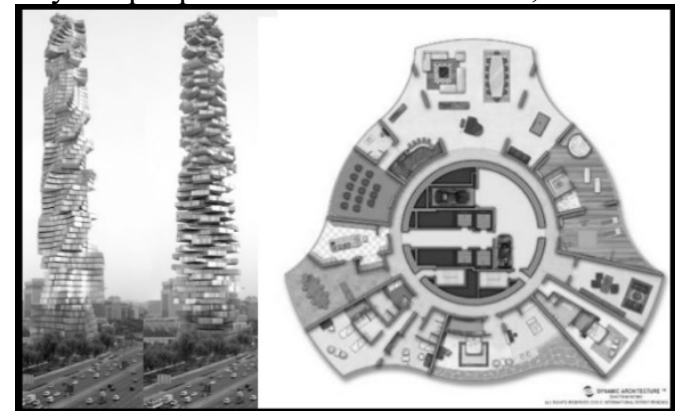

Fig. 11. Dubai’s Moving Skyscraper "Dynamic Tower”.

\section{Teaching Science and Technology Creatively}

To promote creativity in Science and Technology Education in the classroom, we need more learning/teaching strategies. There are four necessary components of teaching for creativity in the classroom.

- Observation: In order to receive knowledge or information through the five senses to develop creativity, observation activities should be introduced [3].

- Curiosity: The best way to make the classroom more meaningful is to arouse curiosity so that the students become inquisitive and eager to learn, or find a way to discover the result by themselves.

- Sense of humor: Many students find the science classroom is boring and difficult. Teachers should introduce a sense of humor through wordplay and other means that will interest the students and make the learning atmosphere more joyful.

- Creativity: As mentioned above, creativity development can make the classroom active and interesting. Active learning in the classroom can help teachers to achieve successful teaching.

Apart from these four components, using new technology, especially IT, is also very important for active learning. Augmented Reality (AR) is a good way of arousing curiosity [5]. Using mathematic tools like Geometer's Sketchpad is also an effective teaching method to make the student understand difficult concepts in mathematics through animation or graphic presentations [6]. 


\section{Conclusion}

In order to develop creativity in science and technology education, teachers play an important role. They have to be good educational facilitators knowing how to manage a classroom in an active way, with methods that build a positive learning atmosphere. The use of SCAMPER methods, as well as the four components of teaching in the classroom-observation, curiosity, sense of humor, and creativity, are the key factors for the development of creativity in science and technology education. Using new technology and IT tools will also help to make teaching/learning science and technology effective and successful.

\section{References}

[1] Anzai, A.: Asia leads global patent applications, with Huawei top, NIKKEI Asia Review, https://asia.nikkei.com/Economy/ (2019)

[2] Yingprayoon, J. International Luncheon on "Teaching Science Creatively Using Locally Produced Low-Cost High-Tech Materials", NSTA Convention, Atlanta, Georgia, USA., $2^{\text {nd }}$ April (2004)

[3] Yew Kam Keong: You are Creative: let your creativity bloom ( $2^{\text {nd }}$ ed.), Mindbloom., Kuala Lumpur, (2001)

[4] Michalko, M. Thinkertoys. : Ten Speed Press, Berkeley, U.S.A. pp. 72-108 (2006)

[5] Yingprayoon, J.: Teaching Mathematics using Augmented Reality. In Proceedings of the 20th Asian Technology Conference in Mathematics, Leshan, China, December 16-20, (2015)

[6] Khairiree, K. (2011) A Study of Constructivist in Mathematics in Virtual Class with Moodle and the Geometer's Sketchpad. In Proceedings of the16th Asian Technology Conference in Mathematics (AIBU, Bolu, Turkey, September 19-23, 2011), ATCM Inc. Published, VA: USA, 97-106. 